TRANSACTIONS OF THE

AMERICAN MATHEMATICAL SOCIETY

Volume 226, 1977

\title{
A DEVELOPMENT OF CONTRACTION MAPPING PRINCIPLES \\ ON HAUSDORFF UNIFORM SPACES
}

BY

\author{
CHENG-MING LEE
}

\begin{abstract}
Certain generalized Banach's contraction mapping principles on metric spaces are unified and/or extended to Hausdorff uniform spaces. Also given are some relationships between the set of all cluster points of the Picard iterates and the set of all fixed points for the mapping. These are obtained by assuming that the latter set is nonempty and by considering certain "quasi"-contractive conditions. The ("quasi") contractive conditions are defined by using a suitable family of pseudometrics on the uniform space.
\end{abstract}

1. Introduction. Let $T$ be a mapping on a nonempty set $X$ into itself. The well-known Banach's contraction mapping principle asserts that if $X$ with a metric $d$ is complete and if

$T$ is a $d$-contraction, i.e., there exists $\lambda \in[0,1)$ such that $d(T(x), T(y)) \leqslant \lambda d(x, y)$ for all $x, y \in X$,

then $T$ has a unique fixed point in $X$ which can be realized as the limit of the Picard iterates $\left\{T^{n}(x)\right\}$ for each $x$ in $X$. An extension of the principle to the general setting for $X$ a Hausdorff uniform space has been recently given by Tan [10, Theorem 2.3] and by Tarafdar [11, Theorem 1.1]. On the other hand, in the metric space setting, many authors have obtained the same conclusions or parts of the conclusions under various conditions which are somehow weaker than condition (B). We propose here to show how some of these results can be unified and/or extended to the general setting. In fact, in \$2, unifying and extending some results of Rakotch [8], Sehgal [9] and Guseman [6], we give a genuine generalization (Theorem 1) of the theorem by Tan and by Tarafdar mentioned above, which has the same conclusions of the classical Banach principle. To conclude, essentially only the existence of a fixed point

Received by the editors June 6, 1975 and, in revised form, November 14, 1975.

AMS (MOS) subject classifications (1970). Primary 47H10; Secondary 54E15.

Key words and phrases. Hausdorff uniform space, family of pseudometrics, topology, uniformity, convergence, cluster point, contraction principle, quasi-contraction. 
for the mapping, the unified and extended sufficient conditions in Theorem 1 are somehow weakened in $\$ 3$, where results (Theorems 2 and 3) are given, extending to the general setting some of the results by Bailey [1], Belluce and Kirk [2], Diaz and Metcalf [3], and Furi and Vignoli [5]. And then in the last section, assuming the existence of fixed points for the mapping, some conclusions of Theorems 1, 2, and 3 are deduced from some even weaker sufficient "quasi" conditions. The results listed as Theorems lq, 2q, and 3q can be viewed as certain extensions and/or variations of some results by Diaz and Metcalf in the interesting paper [3]. Finally, we mention that some relationships of the theorems and some historical aspects of their development will be made clearer by a sequence of remarks in the context.

To fix the general setting to be followed throughout the note, it seems helpful to note that the Banach principle involves not only a topological structure but also a uniformity structure for the basic space, both generated by a single metric on the space. Note also that it is known (see Kelley [7]) that a set $X$ has the structure to be described below if and only if $X$ with a certain uniformity is a Hausdorff uniform space, or if and only if $X$ with a certain topology is a Hausdorff completely regular space. All the terminology for the concepts related to a topology or a uniformity will be those given in the standard book by Kelley [7].

Now, we come to fix the general setting and indicate some simple facts about the setting. Throughout the rest of the note, we use $X$ to denote a nonempty set and $\odot$ a nonempty family of pseudo-metrics on $X$ such that the collection $\left\{S_{p}(x, \gamma): x \in X, p \in \mathcal{P}, \gamma \in(0,+\infty)\right\}$ forms a base for a Hausdorff topology for $X$, where $S_{p}(x, \gamma)$ is the open sphere of $p$-radius $\gamma$ about $x$. Also, $T$ denotes a self-mapping on $X$, and $\mathbf{N}$ the set of all natural numbers. The concepts related to a topology (uniformity, resp.) for $X$ to be concerned will be those related to the topology (uniformity, resp.) generated by $\Phi$ when no specification is given. Sometimes the topology (uniformity, resp.) generated by a single $p$ in $\Phi$ will come into play, in which case, the $p$ will be specified. Note that a sequence, or more generally a net $\left\{x_{i}\right\}$ in $X$, converges to $u$ if and only if it converges to $u$ in $p$-topology (is $p$-Cauchy, resp.) for all $p \in \Phi$, or equivalently the numbers $p\left(x_{i}, u\right)\left(p\left(x_{i}, x_{j}\right)\right.$, resp.) converge to zero for all $p \in \mathcal{P}$. Note also for $x, y \in X, x \neq y$ if and only if there exists $p$ $\in \mathcal{P}$ such that $p(x, y)>0$.

We will also use the following notations:

$$
\begin{aligned}
& O(x, T)=\left\{T^{n}(x): n=0,1,2, \ldots\right\}, \\
& L(x, T)=\left\{y: y \text { is a cluster point of the net }\left\{T^{n}(x): n \in \mathrm{N}\right\},\right. \\
& C(x, T)=O(x, T) \cup L(x, T)
\end{aligned}
$$


Should no ambiguity be possible, we will leave the mapping $T$ unspecified. For convenience, sometimes the set $O(x, T)$ will also be considered as the net $\left\{T^{n}(x): n \in \mathrm{N}\right\}$, where $\mathrm{N}$ is directed by the ordinary order. Note that a point in $L(x)$ must be a limit of a subnet of the net $O(x)$, but it is not necessarily a limit of a subsequence of the sequence $O(x)$. A subnet of the net $O(x)$ will be denoted by $\left\{T^{n_{i}}(x): i \in I\right\}$ or $\left\{T^{n_{j}}(x): j \in J\right\}$, where the directed set $I$ or $J$ is not necessarily a subset of $\mathbf{N}$. The directions for $I, J$ will always be left unspecified. The identiy mapping on $X$ will sometimes also be denoted as $T^{0}$.

\section{A generalized Banach contraction principle.}

THEOREM 1. Suppose that $X$ is sequentially complete (i.e., every Cauchy sequence in $X$ converges) and that the following condition holds:

(BRS) For each $x \in X$ there exists $N=N(x) \in \mathbf{N}$, and for each $p \in \mathcal{P}$ there exists a function $\lambda_{p}:[0,+\infty) \rightarrow[0,1)$ which is monotone decreasing on $(0,+\infty)$ such that for every fixed $x \in X$ the following inequality holds for all $y \in X$ and all $p \in \mathscr{P}$ :

$$
p\left(T^{N(x)}(x), T^{N(x)}(y)\right) \leqslant \lambda_{p}(p(x, y)) p(x, y) .
$$

Then $T$ has a unique fixed point $u$ in $X$ and for each $y \in X, \lim T^{n}(y)=u$.

REMARK 1. This is a unification and extension of some essential results due to Rakotch [8], Sehgal [9] and Guseman [6]. A mapping $T$ satisfying condition (BRS) will be, simply termed $a$ (BRS)-contractive mapping on $X$, in honor of Banach, Rakotch and Sehgal, since in case $\odot$ being a singleton (i.e., $X$ is a metric space) the function $\lambda_{p}$ was introduced by Rakotch and $N(x)$ by Sehgal, both obtained a generalization of the Banach contraction principle. Originally, Sehgal also assumed the continuity of $T$ in his result. The removal of this continuity condition was due to Guseman. Combining and extending their arguments, we present a brief proof of the theorem showing some essential points involved.

Proof OF THEOREM 1. First, note that condition (BRS) implies that for each $x \in X$, and $p \in \mathcal{P}$,

$$
\alpha(x, p) \equiv \sup \left\{p\left(T^{n}(x), x\right): n \in \mathbf{N}\right\} \text { is finite. }
$$

To see this, let

$$
\beta=\max \left\{p\left(T^{k}(x), x\right): k=1,2,3, \ldots, N(x)\right\}
$$

For $n \in \mathbf{N}$ with $n>N(x) \equiv N$, let $s \in \mathbf{N}$ be such that $s N<n \leqslant(s+1) N$, and denote $p_{i}=p\left(T^{n-i N}(x), x\right), k_{i}=\lambda_{p}\left(p_{i}\right)$ for $i=1,2,3, \ldots, s$. Then 


$$
\begin{aligned}
p\left(T^{n}(x), x\right) \leqslant & p\left(T^{N}(x), T^{N}\left(T^{n-N}(X)\right)\right)+p\left(T^{\prime \prime}(x), x\right) \\
\leqslant & k_{1} p_{1}+\beta \leqslant \cdots \\
\leqslant & k_{1} k_{2} k_{3} \cdots k_{i} p_{i} \\
& +\left(k_{1} k_{2} k_{3} \cdots k_{i-1}+\cdots+k_{1} k_{2}+k_{1}+1\right) \beta
\end{aligned}
$$

for all $i$. Denoting $t$ to be the smallest integer $i$ with $p_{i} \leqslant \beta$ (if no such $i$ exists, take $t=s+1$ and $k_{s+1}$ and $p_{s+1}$ below will then be taken as zero), one has $p_{j}>\beta$ for all $j=1,2,3, \ldots, t-1$. Hence by the monotonicity of $\lambda_{p}, k_{j}$ $\left\langle\lambda_{p}(\beta) \equiv k\right.$ for all such $j$. Then

$$
p\left(T^{n}(x), x\right) \leqslant k^{t-1} k_{t} p_{t}+\left(k^{t-1}+\cdots+k^{2}+k+1\right) \beta \leqslant \beta+\beta /(1-k),
$$

which is a finite number independent of $n$. Hence $\alpha(x, p)$ is finite.

Next, applying (1), condition (BRS) implies further that for each $x \in X$,

(2) the sequence $\left\{T^{n}(x)\right\}$ contains a Cauchy subsequence.

In fact, denote $x_{0}=x, m_{0}=N\left(x_{0}\right)$, and inductively define $x_{n}$ $=T^{m_{n-1}}\left(x_{n-1}\right), m_{n}=N\left(x_{n}\right)$ for each $n \in \mathbf{N}$. Then we show that $\left\{s_{n}\right\}$ is the required Cauchy subsequence. It suffices to show that for a given $p \in \Phi$ and a given $\varepsilon>0$,

$$
p\left(x_{n+1}, x_{n+k+1}\right)<\varepsilon \text { for all } k \in \mathbf{N}
$$

when $n$ is large enough. To this end, let $n \in \mathbf{N}$ be fixed and denote $q_{i}=p\left(x_{n-i}, T^{m(k)}\left(x_{n-i}\right)\right)$ and $c_{i}=\lambda_{p}\left(q_{i}\right)$ for $i=0,1,2, \ldots, n$, where $m(k)$ $=m_{n+1}+m_{n+2}+\cdots+m_{n+k}$. Then

$$
\begin{aligned}
p\left(x_{n+1}, x_{n+k+1}\right) & =p\left(T^{m_{n}}\left(x_{n}\right), T^{m_{n}}\left(T^{m(k)}\left(x_{n}\right)\right)\right) \\
& \leqslant c_{0} q_{0} \leqslant \cdots \leqslant c_{0} c_{1} c_{2} \cdots c_{i} q_{i}
\end{aligned}
$$

for $i=0,1,2, \ldots, n$. If $q_{i}<\varepsilon$ for some $i$, then $p\left(x_{n+1}, x_{n+k+1}\right)<\varepsilon$, and we are through. Hence we may assume that $q_{i} \geqslant \varepsilon$ for all $i=0,1,2, \ldots, n$. Then $c_{i} \leqslant \lambda_{p}(\varepsilon) \equiv c$ for all such $i$, and hence

$$
p\left(x_{n+1}, x_{n+k+1}\right) \leqslant c^{n} q_{n} \leqslant c^{n} \alpha(x, p),
$$

which is a number independent of $k$ and is less than $\varepsilon$ if $n$ is large enough since $c \in[0,1)$ and $\alpha(x, p)$ is finite.

Now, since $X$ is sequentially complete, the Cauchy sequence defined in the above proof converges to a point, say $u \in X$. We show that $u$ is in fact a fixed point of $T$. First, note that condition (BRS) implies that

$$
p\left(T^{N(u)}\left(x_{n}\right), T^{N(u)}(u)\right) \leqslant \lambda_{p}\left(p\left(x_{n}, u\right)\right) p\left(x_{n}, u\right) \leqslant p\left(x_{n}, u\right)
$$


for all $p \in \mathcal{P}$, so that $\lim T^{N(u)}\left(x_{n}\right)=T^{N(u)}(u)$. Hence for each $p \in \mathcal{P}$,

$$
p\left(T^{N(u)}(u), u\right)=\lim p\left(T^{N(u)}\left(x_{n}\right), x_{n}\right)
$$

since $p$ is continuous in $X^{2}$. But, by an argument similar to that used in the proof of (2), one shows easily that $\lim p\left(T^{N(u)}\left(x_{n}\right), x_{n}\right)=0$, so that $p\left(T^{N(u)}(u), u\right)=0$ for all $p \in \mathcal{P}$. Hence $T^{N(u)}(u)=u$, i.e., $u$ is a fixed point of $T^{N(u)}$. Then by condition (BRS) again, $u$ is the unique fixed point of $T^{N(u)}$ and, hence, is also the unique fixed point of $T$ since

$$
T^{N(u)}(T(u))=T\left(T^{N(u)}(u)\right)=T(u) .
$$

Finally, $\lim T^{n}(y)=u$ for each $y \in X$. This can be proved by a modification of the argument used in the proof of (2). We omit it here.

REMARK 2. In condition (BRS), if $N(x)=1$ for all $x \in X$ and $\lambda_{p}=\mathrm{a}$ constant for each $p \in \mathcal{P}$, then Theorem 1 reduces to Theorem 2.3 by Tan [10] (cf. also Theorem 1.1 by Tarafdar [11]). Applying his Theorem 2.3, Tan obtained several interesting results. We note that these results either are special cases of Theorem 1 here or can be improved by applying the more general Theorem 1 here. As examples, we mention that his Corollary 2.5 is a special case of Theorem 1 here, and his Corollary 2.6 is another special case of the following corollary to Theorem 1 .

COROLLARY 1.1. Suppose that $X$ is sequentially complete and that there are selfmappings $R, S$ on $X$ such that the composition $R S$ is the identity mapping on $X$ and such that STR is a (BRS)-contractive mapping on $X$ (see Remark 1). Then $T$ has a unique fixed point $u \in X$ and for each $y \in X, R\left(\lim S T^{n} R(y)\right)=u$.

REMARK 3. In Theorem 1, it is not assumed that $T$ or even any of its iterates $T^{n}$ is continuous. It seems interesting to see that condition (BRS) can be weakened or replaced by some other kinds of conditions when the continuity is assumed and/or only parts of the conclusions are to be deduced. Some results of this type will be given in the next two sections. In fact, in \$3, for a continuous mapping to have a fixed point, various sufficient conditions are presented (the conditions are so weak that the net $O(x, T)$ may even have more than one cluster point). To the contrary, in $\$ 4$, the existence of fixed points for the mapping is assumed, and then some of the conditions considered in $\$ \S 2$ and 3 are weakened to ensure that, among others, either $L(x, T) \subseteq F(T)$ or $L(x, T) \cap F(T) \neq \varnothing$, where $F(T)$ is the set of all fixed points for $T$.

3. Existences of fixed points. To obtain the results indicated in Remark 3, we first give a sufficient condition for the set $L(x, T)$ to be nonempty. This involves the notion of the measure of compactness for sets in $X$, which was 
first used by Furi and Vignoli [5] for fixed point theorems in metric spaces.

Definition 1 (cf. [5]). For $A \subseteq X$, the measure of compactness for $A$ is the extended real number $\alpha(A)=\inf \{r: r>0$ and for each $p \in \mathcal{P}$ there exists a finite cover of $A$ by sets of $p$-diameter less than $r$.

Note that by the convention that $\inf (\varnothing)=+\infty, \alpha(A)$ is defined for all $A \subseteq X$. Note also that if $\alpha(A)=0$, then $A$ is totally bounded, and that the closure of a totally bounded set is compact provided that $X$ is complete. (For the notion of total boundedness, see Kelley [7, p. 198].)

Definition 2 (cf. [5]). A mapping $T$ is said to be densifying on $A$ if $\alpha(T(A))<\alpha(A)$ whenever $\alpha(A)>0$.

The following proposition is an easy consequence of the above definitions.

Proposition 1. If $T$ is densifying on $A$ and if $A=T(A) \cup B$, where $B$ is totally bounded, then $A$ is totally bounded.

LeMma 1. If $X$ is complete, and for some $m \in N, T^{m}$ is densifying on $O(x, T)$, then $C(x, T)$ is compact (and hence $L(x, T)$ is nonempty).

Proof. Since $T^{m}(O(x, T)) \cup\left\{T^{k}(x): k=0,1,2, \ldots, m-1\right\}=O(x, T)$, $O(x, T)$ is totally bounded by Proposition 1. But $C(x, T)$ is just the closure of $O(x, T)$. Hence $C(x, T)$ is compact.

REMARK 4. From the conclusion of Theorem 1, one sees easily that a (BRS)contractive mapping $T$ on a complete space $X$ is densifying on $O(x, T)$ for each $x \in X$.

THEOREM 2. Let $X$ be complete, $T$ continuous, and for some $m \in N, T^{m}$ be densifying on $O\left(x_{0}, T\right)$ for a $x_{0} \in X$. If one of the following conditions holds, then $T$ has a fixed point in $C\left(x_{0}, T\right)$, and the fixed point is unique in $X$ if (I) or (II) holds.

(I) There exists a lower semicontinuous function $F: X^{2} \rightarrow(-\infty,+\infty)$ such that for $x, y \in X$ with $x \neq y$,

$$
F\left(T^{N}(x), T^{N}(y)\right)<F(x, y)
$$

for some $N \in \mathbf{N}$.

(II) For $x, y \in X$ with $x \neq y$, there exists $N=N(x, y) \in \mathbf{N}$ such that for all $p \in \mathcal{P}$,

$$
\begin{aligned}
p\left(T^{N}(x), T^{N}(y)\right) & <p(x, y) & & \text { when } p(x, y)>0, \\
& =0 & & \text { when } p(x, y)=0 .
\end{aligned}
$$

(III) For each $x \in X$ with $\delta_{p}(O(x, T))>0$ for some $p \in \mathscr{P}, C(x, T)$ $\neq C\left(T^{N}(x), T\right)$ for some $N \in \mathrm{N}$, where $\delta_{p}(A)$ denotes the $p$-diameter of $A$.

(IV) If $\left\{T^{n_{i}}\left(x_{0}\right): i \in I\right\}$ is a convergent subnet of the net $O\left(x_{0}, T\right)$, then 


$$
\lim p\left(T^{n_{i}}\left(x_{0}\right), T^{n_{i}+1}\left(x_{0}\right)\right)=0 \text { for all } p \in \Phi \text {. }
$$

Remark 5. For $\mathscr{P}=$ a singleton, condition (I) with $N=1$ has been considered by Furi and Vignoli [5], (II) by Bailey [1], and (III) by Belluce and Kirk [2]. (For (IV), cf. [3] and [11], where the asymptotical regularity of $T$ was considered.) We give a proof of the theorem to show the various arguments involved. It seems interesting to note that for the metric space case, condition (I) holds whenever (II) holds, so that in this case, the theorem involving (II) is just a special case of the theorem involving (I). However, in the general setting, whether this is still true requires further investigation (cf. the proof below).

Proof of THEOREM 2. Note first that it is clear that under conditions (I) or (II), $T$ has at most one fixed point in $X$. Hence it remains to show that $T$ does have a fixed point in $C\left(x_{0}\right)=C\left(x_{0}, T\right)$. Next, note that, by Lemma 1, $C\left(x_{0}\right)$ is compact, and, furthermore, $T\left(C\left(x_{0}\right)\right) \subseteq C\left(x_{0}\right)$ since $T$ is continuous.

(i) Suppose that (I) holds. Then the lower semicontinuous function $\varphi$, defined by $\varphi(x)=F(x, T(x))$, assumes a minimum in the compact set $C\left(x_{0}\right)$, say at $u \in C\left(x_{0}\right)$. If $T(u) \neq u$, then by $(\mathrm{I}), \varphi\left(T^{N}(u)\right)<\varphi(u)$ for some $N \in \mathrm{N}$. But $T^{N}(u) \in C\left(x_{0}\right)$, contradicting the minimality of $\varphi(u)$. Hence $T(u)=u$.

(ii) Suppose that (II) holds. Consider the function $F$ defined by $F(x, y)$ $=\sup \{p(x, y): p \in \mathcal{P}\}$. Since each $p \in \mathcal{P}$ is continuous, $F$ is lower semicontinuous. Hence as in (i), the function $\varphi(x)=F(x, T(x))$ assumes a minimum in $C\left(x_{0}\right)$, say at $u \in C\left(x_{0}\right)$. We show that $T(u)=u$ (noting that this does not follow from (i) since whether or not the function $F$ defined here does satisfy the strict inequality in (I) is not clear). Suppose to the contrary that $T(u) \neq u$. Then $\varphi(u)>0$. Denote $N_{0}=0, \mathscr{P}_{1}=\left\{p: p \in \mathcal{P}\right.$ and $p\left(T^{N_{0}}(u), T^{N_{0}+1}(u)\right)$ $>0$ \}, and inductively for $n \in \mathrm{N}$ define $N_{n}$ to be the smallest integer such that

$$
p\left(T^{N_{n}}(u), T^{N_{n}+1}(u)\right)<p\left(T^{N_{n-1}}(u), T^{N_{n-1}+1}(u)\right)
$$

for all $p \in \mathscr{P}_{n}$, and $\mathscr{P}_{n+1}=\left\{p: p \in \mathcal{P}\right.$ and $\left.p\left(T^{N_{n}}(u), T^{N_{n}+1}(u)\right)>0\right\}$. Then note first that for $n \in \mathrm{N}$, if $k \leqslant N_{n}$, then

$$
p\left(T^{k}(u), T^{k+1}(u)\right) \geqslant p\left(T^{N_{n-1}}(u), T^{N_{n-1}+1}(u)\right)
$$

for all $p \in \mathcal{P}$. Now, since $C\left(x_{0}\right)$ is compact, the net $\left\{T^{N_{n}}(u): n \in \mathrm{N}\right\}$ in $C\left(x_{0}\right)$

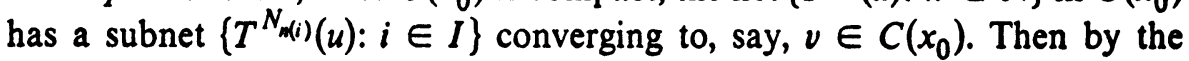
continuity of $T$ and $p \in \mathcal{P}$, one has

$$
p\left(T^{k}(v), T^{k+1}(v)\right)=\lim p\left(T^{k+N_{n(i)}}(u), T^{k+N_{m(i)}+1}(u)\right)
$$

for all $p \in \mathscr{P}$ and for all $k=0,1,2, \ldots$ And then using (3), one shows that 


$$
p\left(T^{k}(v), T^{k+1}(v)\right) \geqslant p(v, T(v))
$$

for all $p$ and $k$ involved. This, by (II), is possible only when $p(v, T(v))$ $=0$ for all $p \in \mathcal{P}$. But then $\varphi(v)=0<\varphi(u)$, contradicting the minimality of $\varphi(u)$ since $v \in C\left(x_{0}\right)$. Hence one must have $T(u)=u$.

(iii) Suppose (III) holds. Consider the family of sets $\mathscr{F}=\{K: K$ is a nonempty closed subset of $C\left(x_{0}\right)$ and $T(K) \subseteq K$, which is nonempty since $T\left(C\left(x_{0}\right)\right) \subseteq C\left(x_{0}\right)$. Partially order $\mathscr{F}$ by inclusion. Then by the compactness of $C\left(x_{0}\right)$, one can apply Zorn's lemma and obtain a minimal element $K_{0}$ in $\Phi_{5}$ Let $u \in K_{0}$;one has by the minimality of $K_{0}$ that $C(u, T)=K_{0}$. Suppose that $K_{0}$ contains another point $v$. Then there exists $p \in \Phi$ with $p(u, v)>0$, so that

$$
\delta_{p}(O(u, T))=\delta_{p}\left(K_{0}\right)>0 .
$$

Then by (III), $C(u, T) \neq C\left(T^{N}(u), T\right)$ for some $N \in \mathrm{N}$. This contradicts the minimality of $K_{0}$ since $C\left(T^{N}(u), T\right) \in \mathscr{F}$ and $C\left(T^{N}(u), T\right) \subseteq C(u, T)$ $=K_{0}$. Hence $K_{0}$ contains only one point $u$ and hence it is a fixed point of $T$.

(iv) Suppose (IV) holds. Then each $u \in L\left(x_{0}, T\right)$ (which is nonempty) is a fixed point of $T$. In fact, for $u \in L\left(x_{0}\right)$, converging to $u$ there is a subnet $\left\{T^{n_{1}}\left(x_{0}\right): i \in I\right\}$ of the net $O\left(x_{0}, T\right)$. Then by the continuity of $T$ and $p \in \mathcal{P}$ and by (IV), one has

$$
p(u, T(u))=\lim p\left(T^{n_{i}}\left(x_{0}\right), T^{n_{i}+1}\left(x_{0}\right)\right)=0
$$

for all $p \in \mathcal{P}$, so that $T(u)=u$, completing the proof.

Corollary 2.1. Let $X$ be complete, and for some $m \in \mathrm{N}, n \in \mathrm{N}, T^{n}$ be continuous and $T^{m}$ be densifying on $O\left(x_{0}, T^{n}\right)$ for $a x_{0} \in X$. If one of the following conditions holds, then $T$ has a fixed point in $C\left(x_{0}, T^{n}\right)$, and the fixed point is unique in $X$.

(I)s There exists a lower semicontinuous function $F: X^{2} \rightarrow(-\infty,+\infty)$ such that for $x, y \in X$ with $x \neq y$,

$$
F\left(T^{n N}(x), T^{n N}(y)\right)<F(x, y)
$$

for some $N \in \mathbf{N}$.

(II)s For $x, y \in X$ with $x \neq y$, there exists $N \in \mathrm{N}$ such that for all $p \in \Phi$,

$$
\begin{aligned}
p\left(T^{n N}(x), T^{n N}(y)\right) & <p(x, y) & & \text { when } p(x, y)>0, \\
& =0 & & \text { when } p(x, y)=0 .
\end{aligned}
$$

Proof. Applying Theorem 2 to the mapping $T^{n}$, one concludes that in $X, T^{n}$ has a unique fixed point $u \in C\left(x_{0}, T^{n}\right)$. Then it follows that $u$ is also the unique fixed point for $T$. 
REMARK 6. The conditions involving the continuity and densifyingness in Theorem 2 are thus weakened (when $n>1$ ) in the corollary, while conditions (I) and (II) are strengthened to (I)s and (II)s. On the other hand, replacing the continuity condition by the stronger condition of being 9 -nonexpansive, we can weaken the other conditions. In fact, we have the following result.

THEOREM 3. Let $X$ be complete, and for some $m \in N, T^{m}$ be densifying on

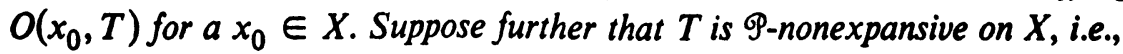

$$
p(T(x), T(y)) \leqslant p(x, y) \text { for all } x, y \in X \text { and all } p \in \mathcal{P} \text {. }
$$

If one of the following conditions holds, then $\lim T^{n}\left(x_{0}\right)$ exists and is a fixed point of $T$.

(II)w For $x, y \in X$ with $x \neq y$, there exist $p \in \mathscr{P}, N \in \mathbf{N}$ such that

$$
p\left(T^{N}(x), T^{N}(y)\right)<p(x, y) .
$$

(III)w For each $x \in X$, if $O(x, T)$ contains more than one point, then there exists $p \in \mathscr{P}$ such that $T$ is not p-isometric on $O(x, T)$, i.e., there are some nonnegative integers $M, N, K$ such that

$$
p\left(T^{M+K}(x), T^{N+K}(x)\right) \neq p\left(T^{M}(x), T^{N}(x)\right) .
$$

(IV)w There exists a convergent subnet $\left\{T^{n_{i}}\left(x_{0}\right): i \in I\right\}$ of the net $O\left(x_{0}, T\right)$ such that

$$
\lim p\left(T^{n_{i}}\left(x_{0}\right), T^{n_{i}+1}\left(x_{0}\right)\right)=0 \text { for all } p \in \Phi .
$$

REMARK 7. It is sufficient to assume that $L\left(x_{0}, T\right)$ is nonempty instead of the conditions involving completeness and densifyingness. Doing this, the theorem involving (II)w has been obtained by Tan [10] and that involving (III)w is just an extension of Theorem 2.2 in [2] by Belluce and Kirk. The part of the proof involving (IV)w is trivial, while the other parts follow immediately from the following result, which can be deduced easily from Theorem 1 in [4] by Edelstein. We omit all the proof here.

THEOREM A. If $T$ is $\odot$-nonexpansive on $X$ and $u \in L\left(x_{0}, T\right)$ for a $x_{0} \in X$, then $T$ is p-isometric on $O(u, T)$ for all $p \in \mathcal{P}$, i.e.,

$$
p\left(T^{M+K}(u), T^{N+K}(u)\right)=p\left(T^{M}(u), T^{N}(u)\right)
$$

for all nonnegative integers $M, N, K$ and for all $p \in \mathcal{P}$.

4. Quasi-contractive mappings. Let $F(T)$ be the set of all fixed points of $T$ in $X$ and suppose that it is nonempty. In this section, contrast the contractive mappings (i.e., mappings which shrink "distances" of points in some manner 
like (BRS), (II), etc.) considered in the previous two sections, we will consider their corresponding quasi-contractive mappings (by which we mean mappings which shrink distances between points and the set $F(T)$ ).

For $A \subseteq X, p \in \mathcal{P}$, define for each $x \in X$,

$$
p(x, A)=\inf \{p(x, a): a \in A\} .
$$

Then one shows easily that $p(\cdot, A)$ is continuous in $X$. Note also that a point $u \in X$ is in the closure of $A$ if and only if $p(u, A)=0$ for all $p \in \Phi$.

The following lemma, similar to Theorem $A$ in the last section, is our starting point.

Lemar 2. Suppose that $T$ is 9 -quasi-nonexpansive on $X$ with respect to $F(T)$, i.e., for all $x \in X$ and $p \in \mathcal{P}$,

$$
p(T(x), F(T)) \leqslant p(x, F(T)) .
$$

Then for $u, v \in L(x, T)$,

$$
p(u, F(T))=p(\nu, F(T)) \text { for all } p \in \Phi .
$$

Proof. The sequence $\left\{p\left(T^{n}(x), F(T)\right)\right\}$, being monotone decreasing by (4), converges to a nonnegative number, say $\alpha$. Now, of the net $\left\{T^{n}(x): n \in \mathrm{N}\right\}$, let $\left\{T^{n_{1}}(x): i \in I\right\}$ be a subnet convergent to $u$. Then $\left\{p\left(T^{n_{1}}(x), F(T)\right): i \in I\right\}$ is a subnet of the convergent net $\left\{p\left(T^{n}(x), F(T)\right)\right\}$, and hence must converge to $\alpha$, too. It then follows from the continuity of $p(\cdot, F(T))$ that $p(u, F(T))=\alpha$. Similarly, $p(v, F(T))=\alpha$.

THEOREM 3q. Suppose that $T$ is continuous and 9 -quasi-nonexpansive on $X$ with respect to $F(T)$. If one of the following conditions holds, then $L(x, T) \subseteq F(T)$.

(II)wq For each $y \notin F(T)$, there exists $p \in \Phi$ and $N \in \mathbf{N}$ such that $p\left(T^{N}(y), F(T)\right) \neq p(y, F(T))$.

(IV)w There exists a convergent subnet $\left\{T^{n_{1}}(x): i \in I\right\}$ of the net $\left\{T^{n}(x): n\right.$ $\in \mathrm{N}\}$ such that $\lim p\left(T^{n_{1}}(x), T^{n_{i}+1}(x)\right)=0$ for all $p \in \Phi$.

Proof. Let $u \in L(x, T)$. We show that $u \in F(T)$. If (II)wq holds and $u \notin F(T)$, then there exist $p_{0} \in \mathcal{P}$ and $N \in \mathrm{N}$ such that

$$
p_{0}\left(T^{N}(u), F(T)\right) \neq p_{0}(u, F(T)) .
$$

But since $T$ is continuous, $T^{k}(u) \in L(x, T)$ for all $k \in \mathrm{N}$, and hence by Lemma 2, $p\left(T^{k}(u), F(T)\right)=p(u, F(T))$ for all $p \in \mathcal{P}$ and $k \in N$, which contradicts (5). Hence $u \in F(T)$. If (IV)w holds, then $\lim T^{n_{1}}(x)=v$ $\in L(x, T)$ and then 


$$
p(v, T(v))=\lim p\left(T^{n_{i}}(x), T^{n_{i}+1}(x)\right)=0 \text { for all } p \in \mathcal{P}
$$

since both $p$ and $T$ are continuous. Hence $T(v)=v$, i.e., $v$ $\in F(T)$ and hence $p(v, F(T))=0$ for all $p \in \Phi$. Then it follows from Lemma 2 that $p(u, F(T))=0$ for all $p \in \Phi$ since both $u, v \in L(x, T)$. Therefore $u$ is in the closure of $F(T)$, and hence in $F(T)$ since $F(T)$ is closed by the continuity of $T$.

RemarK 8. Note that under condition (II)wq, the set $L(x, T)$ may be empty. One way to ensure that $L(x, T)$ is nonempty is to assume that $X$ is complete and that $T^{m}$ is densifying on $O(x, T)$ (see Lemma 1 in $\$ 3$ ). It seems worthwhile to note that under the additional assumptions, one has that the sequence $T^{n}(x)$ is convergent when $L(x, T)$ is a singleton. In fact, if $L(x, T)$ contains only one point, say $u$, then $\lim T^{n}(x)=u$. For otherwise, there would be a subnet $\left\{T^{n_{1}}(x): i \in I\right\}$ of the net $\left\{T^{n}(x): n \in \mathrm{N}\right\}$ such that $\left\{T^{n_{1}}(x)\right\}$ has no subnet converging to $u$. But as a net in the compact set $C(x, T),\left\{T^{n_{i}}(x)\right\}$ has a subnet converging to a point $v \in L(x, T)$. But then $u \neq v$, a contradiction. On the other hand, even without the additional assumptions, one sees easily that $L(x, T)$ must lie on the boundary of $F(T)$ when $L(x, T)$ contains more than one point. For further structures of the set $L(x, T)$ under various other canditions, see Diaz and Metcalf [3] and Tarafdar [11].

Remark 9. From the last part in the proof of Theorem 3q, one sees that " $L(x, T) \subseteq F(T)$ " is an immediate consequence of the condition " $L(x, T)$ $\cap F(T) \neq \varnothing$ " provided that $T$ is continuous and $\mathcal{P}$-quasi-nonexpansive. The following results, parallel to Theorems 2 and 1 , give sufficient conditions for $L(x,) \cap F(T) \neq \varnothing$.

TheOrem 2q. Let $X$ be complete, $T$ continuous, and for a $m \in \mathbf{N}, T^{m}$ densifying on $O(x, T)$. If $F(T)$ is nonempty and

(II)q for each $y \notin F(T)$ there exists $N \in \mathbf{N}$ such that for all $p \in \mathcal{P}$,

$$
\begin{aligned}
p\left(T^{N}(y), F(F)\right) & <p(y, F(T)) & & \text { when } p(y, F(T))>0, \\
& =0 & & \text { when } p(y, F(T))=0,
\end{aligned}
$$

then $L(x, T) \cap F(T) \neq \varnothing$.

Proof. If $T^{k}(x) \in F(T)$ for a nonnegative integer $k$, then $T^{k}(x) \in$ $L(x, T) \cap F(T)$. Suppose that $T^{k}(x) \notin F(T)$ for $k=0,1,2,3, \ldots$. Similar to (ii) in the proof of Theorem 2, let $N_{0}=0$ and $\mathscr{P}_{0}=\{p: p \in \Phi$ with $\left.p\left(T^{N_{0}}(x), F(T)\right)>0\right\}$, and inductively define for each $n \in \mathrm{N}, N_{n}$ to be the smallest integer such that

$$
p\left(T^{N_{n}}(x), F(T)\right)<p\left(T^{N_{n-1}}(x), F(T)\right) \text { for all } p \in \mathcal{P}_{n-1}
$$

and $\mathscr{P}_{n}=\left\{p: p \in \mathcal{P}\right.$ with $\left.p\left(T^{N_{n}}(x), F(T)\right)>0\right\}$. Then for $t \leqslant N_{n}$, one has 


$$
p\left(T^{t}(x), F(T)\right) \geqslant p\left(T^{N_{n-1}}(x), F(T)\right) \text { for all } p \in \mathcal{P} .
$$

Now, since $C(x, T)$ is compact, the net $\left\{T^{N_{n}}(x): n \in \mathrm{N}\right\}$ contains a convergent subnet $\left\{T^{N_{n}(i)}(x): i \in I\right\}$, say converging to $u \in L(x, T) \subseteq C(x, T)$. By the continuity of $p(\cdot, F(T))$,

$$
p\left(T^{t}(u), F(T)\right)=\lim p\left(T^{t+N_{(i)}}(x), F(T)\right)
$$

for all $p \in \Phi$ and all $t=0,1,2, \ldots$. Hence it follows from (6) that $p\left(T^{t}(u), F(T)\right) \geqslant p(u, F(T))$ for all such $p$ and $t$. Then (II)q implies that $p^{\prime}(u, F(T))=0$ for all $p \in \Phi$. Hence $u$ is in the closure of $F(T)$ and hence in $F(T)$ since $F(T)$ is closed.

THEOREM 1q . Let $F(T)$ be nonempty and compact. Suppose that the following condition holds:

(BRS)q For each $p \in \Phi$ there exists a function $\lambda_{p}:[0,+\infty) \rightarrow[0,1)$ which is monotone decreasing on $(0,+\infty)$ and for each $y \in X \sim F(T)$, there exists $N=N(y) \in \mathbf{N}$ such that

$$
p\left(T^{N}(y), F(T)\right) \leqslant \lambda_{p}(p(y, F(T))) p(y, F(T)) .
$$

Then for each $x \in X$, the sequence $\left\{T^{n}(x): n \in \mathrm{N}\right\}$ has a subsequence converging to a fixed point of $T$ provided that either $\Phi$ is a singleton or $F(T)$ is a singleton.

Proof. Let $x \in X$ be fixed and define $x_{0}=x, m_{0}=N\left(x_{0}\right)$ and inductively $x_{n}=T^{m_{n-1}}\left(x_{n-1}\right), m_{n}=N\left(x_{n}\right)$ for $n \in N$. Then modifying parts of the argument in the proof of Theorem 1 , one shows that $\lim p\left(x_{n}, F(T)\right)=0$ for all $p \in \Phi$. Hence it follows that $\lim x_{n}=u$ provided that $u$ is the only element in $F(T)$. If it is that $P$ is a singleton, say $\mathcal{P}=\{d\}$ (i.e., $X$ is a metric space), then by the compactness of $F(T)$, the continuous function $d\left(x_{n}, \cdot\right)$ assumes the value $d\left(x_{n}, F(T)\right)$ in $F(T)$, say at $u_{n} \in F(T)$ for each $n$. Then

$$
\lim d\left(x_{n}, u_{n}\right)=\lim d\left(x_{n}, F(T)\right)=0 .
$$

Without loss of generality since $F(T)$ is compact, we may assume that $\left\{u_{n}\right\}$ converges to an element $u \in F(T)$. Then

$$
d\left(x_{n}, u\right) \leqslant d\left(x_{n}, u_{n}\right)+d\left(u_{n}, u\right),
$$

so that $\lim x_{n}=u \in F(T)$, completing the proof.

REMARK 10. It would be interesting to see when the condition that either $\Phi$ or $F(T)$ is a singleton can be removed from Theorem lq.

\section{REFERENCES}

1. D. F. Bailey, Some theorems on contractive mappings, J. London Math. Soc. 41 (1966), 101-106. MR 32 \#6434. 
2. L. P. Belluce and W. A. Kirk, Some fixed point theorems in metric and Banach spaces, Canad. Math. Bull. 12 (1969), 481-491. MR 40 \#3388.

3. J. B. Diaz and F. T. Metcalf, On the set of subsequential limit points of successive approximations, Trans. Amer. Math. Soc. 135 (1969), 459-485. MR 38 \#2644.

4. M. Edelstein, On nonexpansive mappings of uniform spaces, Nederl. Akad. Wetensch. Proc. Ser. A 68 = Indag. Math. 27 (1965), 47-51. MR 30 \#2461.

5. M. Furi and A. Vignoli, $A$ fixed point theorem in complete metric spaces, Bull. Un. Math. Ital. (4) 2 (1969), 505-509. MR 41 \# 1034.

6. L. F. Guseman, Jr., Fixed point theorems for mappings with a contractive iterate at a point, Proc. Amer. Math. Soc. 26 (1970), 615-618. MR 42 \#19.

7. J. L. Kelley, General topology, Van Nostrand, Princeton, N. J., 1955. MR 16, 1136.

8. E. Rakotch, A note on contractive mappings, Proc. Amer. Math. Soc. 13 (1962), 459-465. MR 26 \#5555.

9. V. M. Sehgal, $A$ fixed point theorem for mappings with a contractive iterate, Proc. Amer. Math. Soc. 23 (1969), 631-634. MR 40 \#3531.

10. K.-K. Tan, Fixed point theorems for nonexpansive mappings, Pacific J. Math. 41 (1972), 829-842. MR 47 \#2454.

11. E. Tarafdar, An approach to fixed-point theorems on uniform spaces, Trans. Amer. Math. Soc. 191 (1974), 209-225. MR 50 \# 14725.

Department of Mathematics, University of Wisconsin, Milwaukee, Wisconsin 53201 